\title{
Ipilimumab Real-World Efficacy and Safety in Korean Melanoma Patients from the Korean Named-Patient Program Cohort
}

\author{
Minkyu Jung, $\mathrm{MD}^{1}$ \\ Jeeyun Lee, MD, PhD² \\ Tae Min Kim, MD, PhD ${ }^{3}$ \\ Dae Ho Lee, MD, PhD ${ }^{4}$ \\ Jin Hyung Kang, MD, PhD \\ Sung Young Oh, MD, $\mathrm{PhD}^{6}$ \\ Soo Jung Lee, MD, $\mathrm{PhD}^{7}$ \\ Sang Joon Shin, MD, $\mathrm{PhD}{ }^{1}$
}

${ }^{1}$ Division of Medical Oncology, Department of Internal Medicine, Yonsei Cancer Center, Yonsei University College of Medicine, Seoul, ${ }^{2}$ Division of Hematology-Oncology, Department of Medicine, Samsung Medical Center, Sungkyunkwan University School of Medicine, Seoul, ${ }^{3}$ Department of Internal Medicine, Seoul National University Hospital, Seoul National University College of Medicine, Seoul, ${ }^{4}$ Department of Oncology, Asan Medical Center, University of Ulsan College of Medicine, Seoul, ${ }^{5}$ Division of Hematology-Oncology, Department of Internal Medicine, Seoul St. Mary's Hospital, College of Medicine, The Catholic University of Korea, Seoul, ${ }^{6}$ Department of Internal Medicine, Dong-A University Hospital, Dong-A University College of Medicine, Busan, ${ }^{7}$ Division of

Hematology-Oncology, Department of Internal Medicine, Kyungpook National University Hospital, Kyungpook National University School of Medicine, Daegu, Korea

Correspondence: Sang Joon Shin, MD, PhD Division of Medical Oncology, Department of Internal Medicine, Yonsei Cancer Center, Yonsei University College of Medicine, 50 Yonsei-ro, Seodaemun-gu, Seoul 03722, Korea

Tel: $82-2-2228-8138$

Fax: 82-2-393-3652

E-mail: SSJ338@yuhs.ac

Received January 18, 2016

Accepted April 6, 2016

Published Online April 27, 2016

${ }^{*}$ Minkyu Jung and Jeeyun Lee contributed equally to this work.

\section{Purpose}

Ipilimumab improves survival in advanced melanoma patients. However, the efficacy and safety of ipilimumab has not been evaluated in Asian melanoma patients with a high frequency of mucosal and acral melanoma subtypes.

\section{Materials and Methods}

Advanced melanoma patients treated with $3 \mathrm{mg} / \mathrm{kg}$ ipilimumab in a Korean multicenter named-patient program (NPP) were evaluated between September 2014 and July 2015. Baseline characteristics and blood parameters including neutrophil to lymphocyte ratio (NLR) were assessed, and outcome and adverse events were evaluated according to subtypes.

\section{Results}

A total of 104 advanced melanoma patients were treated. The primary sites were acral (31.7\%), mucosal (26\%), cutaneous (26\%), uveal (9.6\%), and unknown (6.7\%). Sixty-eight patients (65.4\%) experienced adverse events, and the most common toxicity was skin rash (22.1\%), 10 patients (9.6\%) experienced adverse events of grade 3 or higher. The median progression-free survival (PFS) was 2.73 months (95\% confidence interval, 2.67 to 2.85 ), and there was no difference in PFS according to subtypes. Poor performance status, liver metastasis, and NLR $(\geq 5)$ were independent poor prognostic factors by multivariate analysis.

\section{Conclusion}

In the Korean NPP cohort, ipilimumab showed similar efficacy and tolerability compared to Western patients, regardless of subtypes. All subtypes should benefit from ipilimumab with consideration of performance status, liver metastasis, and NLR.

\section{Key words}

Melanoma, Immunotherapy, Ipilimumab, Biomarkers 


\section{Introduction}

Malignant melanoma is one of the most aggressive cancers and its incidence has shown a rapid increase [1-3]. Many treatments for melanoma, including chemotherapies, immunotherapies, and combined biochemotherapy regimens have been utilized but have failed to significantly improve outcome and the median overall survival (OS) of metastatic melanoma is approximately 8 months [4]. However, studies reported in recent years have resulted in many breakthroughs in the melanoma field. Two extraordinary advances were recently achieved with positive results from two separate studies of new therapies, ipilimumab [5] in immunotherapy and vemurafenib [6] in molecular targeted therapy.

Ipilimumab (Yervoy, Bristol-Myers Squibb) is a fully human monoclonal antibody against the cytotoxic T-lymphocyte-associated antigen 4 receptor. In phase III clinical trials, treatment with ipilimumab resulted in a statistically significant improvement in OS of patients with metastatic melanoma [5]. A pooled analysis of 1,861 patients treated with ipilimumab showed long-term survival with a 3-year survival rate of $22 \%$ [7]. In addition, many expanded access programs (EAP) in various countries reported on the benefit of ipilimumab in a real world setting [8]. Compared to Western countries, the incidence of melanoma in Eastern counties is very low but has also increased [9]. The most common subtype in Western countries is cutaneous melanoma, while acral and mucosal melanoma are the most common subtypes in Eastern countries [9-12]. Ipilimumab has been approved in Asian countries including Korea; however, data on the efficacy and safety of ipilimumab in Asian patients with melanoma were limited.

Therefore, we reported the outcomes of ipilimumab in Korean patients enrolled in the named-patient program (NPP) cohort. In addition, we examined the association between response to ipilimumab and melanoma subtype, $B R A F$ mutation, and blood parameters.

\section{Materials and Methods}

\section{Patients' inclusion and treatment plan}

Patients who met the following eligibility criteria were enrolled in the ipilimumab NPP; a histologically confirmed stage IV malignant melanoma according to the American Joint Committee on Cancer (AJCC), and Eastern Cooperative Oncology Group (ECOG) performance status (PS) 0, 1, or 2. All patients received four cycles of $3 \mathrm{mg} / \mathrm{kg}$ ipilimumab every 3 weeks, unless severe adverse events (AEs) or rapid progression of disease occurred. M-stage was defined according to site metastasis in combination with elevated lactate dehydrogenase (LDH) levels, as described previously [13]. Records of all patients who received at least one dose of ipilimumab were reviewed retrospectively. The study protocol was approved by the ethics committee from all six hospitals and this study was conducted according to the Declaration of Helsinki.

\section{Data collection}

The medical records of eligible patients were retrieved and included age, sex, ECOG PS, previous therapies, melanoma subtype and disease stage, metastatic site, LDH, and BRAF and $c$-KIT mutational status if available. Treatment-related AEs using the Common Terminology Criteria for Adverse Events ver. 4.0; hematological and serum parameters including absolute neutrophil count (ANC), and lymphocyte count; date of progression; and reason for cessation of ipilimumab (progressive disease, AE, and death) were also collected. Patients were reviewed routinely every 3 weeks during treatment and every 8 weeks during follow-up. Radiological imaging was generally performed 6-9 weeks after ipilimumab induction and every 8 weeks thereafter. Responses were scored according to Response Evaluation Criteria in Solid Tumor (RECIST) ver. 1.1 criteria.

\section{Statistical analysis}

Baseline, pre-treatment white blood cell count, ANC, absolute lymphocyte count (ALC), neutrophil to lymphocyte ratio (NLR), and serum LDH were analyzed. Progressionfree survival (PFS) was defined as the time from the start of ipilimumab to the onset of progression or death. Patients without progression and still alive at the time of analysis were censored. OS was defined as the time from the start of ipilimumab to death from any cause. Univariate analysis for clinical and laboratory parameters was performed with respect to OS and PFS. Survival curves of categorical variables were calculated using the Kaplan-Meier method and compared using the log-rank test. Continuous variables in association with survival outcomes were examined by means of martingale residuals. Multivariate models of patient and tumor characteristics in association with PFS and OS were based on Cox proportional hazards regression analyses. Two-tailed $\mathrm{p}<0.05$ was considered statistically significant for all analyses. SPSS ver. 15.0 (SPSS Inc., Chicago, IL) was used for data analysis. 


\section{Results}

\section{Patient characteristics}

Between September 2014 and July 2015, 104 patients were treated with ipilimumab at six Korean hospitals. Patients' characteristics are summarized in Table 1 . The median age of patients was 58 years (interquartile range, 50 to 66 ) and 51 patients (49\%) were male. The most common subtype was acral melanoma (33 patients, $31.7 \%), 27$ patients $(26 \%)$ had mucosal melanoma, and 27 patients $(26 \%)$ had skin melanoma. All 104 patients had AJCC stage IV melanoma, and the majority of patients were stage M1c (76\%). Sixty-two patients $(65.3 \%)$ had elevated baseline $\mathrm{LDH}$ and 20 patients $(19.2 \%)$ had NLR $\geq 5$. BRAF and $c-K I T$ mutations were evaluated in 87 and 54 patients, respectively, and the incidence of BRAF and c-KIT mutations was $18.4 \%$ and $13.0 \%$, respectively. Approximately $40 \%$ of patients received ipilimumab as first line treatment, and $28 \%$ patients had received at least three prior systemic therapies, median number of prior systemic treatments was two (range, 1 to 5). Sixty-nine patients $(66.3 \%)$ received all four doses of ipilimumab, and nine patients received three doses, 11 patients received two doses, and 15 patients received one dose. The most common reason for drug discontinuation was rapid disease progression.

\section{Safety}

Of all patients, 68 patients $(65.4 \%)$ experienced treatment related AEs of any grade (Table 2), and the most common toxicities were pruritus $(31.7 \%)$ and skin rash $(22.1 \%)$. The majority of treatment related AEs were grade 1 or 2, and were generally manageable and reversible without sequelae. Ten patients (9.6\%) experienced grade 3 toxicities, and seven patients experienced treatment related AEs, and three cases were considered to be disease related events. One patient suffered from grade 3 fatigue and grade 2 nausea after two cycles of ipilimumab and brain metastasis was detected on brain magnetic resonance imaging. One patient developed grade 3 elevation of aspartate aminotransferase (AST)/alanine aminotransferase (ALT) after three cycles of ipilimumab and showed progression of liver metastasis according to RECIST ver. 1.1 criteria. One patient complained of grade 3 generalized pain, which improved to grade 1 after morphine treatment. No patient experienced grade 4 or $5 \mathrm{AEs}$, and there were no immune response related serious AEs.
Table 1. Baseline characteristics

\begin{tabular}{|c|c|}
\hline Variable & No. $(\%)$ \\
\hline Age, median (IQR, yr) & $58(50-66)$ \\
\hline \multicolumn{2}{|l|}{ Sex } \\
\hline Male & $51(49)$ \\
\hline Female & $53(51)$ \\
\hline \multicolumn{2}{|l|}{ ECOG PS } \\
\hline 0 & $28(26.9)$ \\
\hline 1 & $64(61.5)$ \\
\hline 2 & $12(11.5)$ \\
\hline \multicolumn{2}{|l|}{ Primary site } \\
\hline Acral & $33(31.7)$ \\
\hline Mucosal & $27(26)$ \\
\hline Cutaneous & $27(26)$ \\
\hline Uveal & $10(9.6)$ \\
\hline Primary origin unknown & $7(6.7)$ \\
\hline \multicolumn{2}{|l|}{ M stage (LDH included) } \\
\hline M1a & $17(16.3)$ \\
\hline M1b & $8(7.7)$ \\
\hline M1c & $79(76)$ \\
\hline \multicolumn{2}{|l|}{ Patients with liver metastasis } \\
\hline Yes & $35(33.7)$ \\
\hline No & $69(66.3)$ \\
\hline \multicolumn{2}{|l|}{ Patients with brain metastasis } \\
\hline Yes & $13(12.5)$ \\
\hline No & $91(87.5)$ \\
\hline \multicolumn{2}{|l|}{ Elevated LDH (n=95) } \\
\hline Yes & $62(65.3)$ \\
\hline No & $33(32.7)$ \\
\hline \multicolumn{2}{|c|}{ Neutrophil to lymphocyte ratio } \\
\hline$<5$ & $84(80.8)$ \\
\hline$\geq 5$ & $20(19.2)$ \\
\hline \multicolumn{2}{|l|}{$B R A F$ mutation $(\mathrm{n}=87)$} \\
\hline Mutant & $16(18.4)$ \\
\hline Wild type & $71(71.6)$ \\
\hline \multicolumn{2}{|l|}{$c-K I T$ mutation $(\mathrm{n}=54)$} \\
\hline Mutant & $7(13)$ \\
\hline Wild type & $47(87)$ \\
\hline \multicolumn{2}{|l|}{ No. of previous therapies } \\
\hline 1 & $41(39.4)$ \\
\hline 2 & $34(32.7)$ \\
\hline$\geq 3$ & $29(27.9)$ \\
\hline
\end{tabular}

IQR, interquartile range; ECOG PS, Eastern Cooperative Oncology Group performance status; LDH, lactate dehydrogenase. 
Table 2. Treatment-related adverse events

\begin{tabular}{|c|c|c|c|c|}
\hline \multirow{2}{*}{ Adverse event } & \multicolumn{4}{|c|}{ No. $(\%)(n=104)$} \\
\hline & Grade 1 & Grade 2 & Grade 3 & Grade 4 \\
\hline \multicolumn{5}{|l|}{ Immune related } \\
\hline Diarrhea & $6(5.8)$ & $1(1.0)$ & 0 & 0 \\
\hline Rash & $17(16.3)$ & $5(4.8)$ & $1(1.0)$ & 0 \\
\hline Pruritus & $14(13.5)$ & $18(17.3)$ & $1(1.0)$ & 0 \\
\hline Thyroiditis & $1(1.0)$ & $1(1.0)$ & 0 & 0 \\
\hline \multicolumn{5}{|l|}{ Non-immune related } \\
\hline Nausea & $7(6.7)$ & $4(3.8)$ & $1(1.0)$ & 0 \\
\hline Vomiting & $3(2.9)$ & $2(1.9)$ & 0 & 0 \\
\hline Fatigue & $13(12.5)$ & $6(5.8)$ & $3(2.9)$ & 0 \\
\hline Pain & $8(7.7)$ & $7(6.7)$ & $1(1.0)$ & 0 \\
\hline Dyspnea & $2(1.9)$ & $4(3.8)$ & 0 & 0 \\
\hline Edema & $2(1.9)$ & 0 & $1(1.0)$ & 0 \\
\hline Elevated AST / ALT & 0 & 0 & $1(1.0)$ & 0 \\
\hline Anemia & 0 & $1(1.0)$ & $1(1.0)$ & 0 \\
\hline
\end{tabular}

AST, aspartate aminotransferase; ALT, alanine aminotransferase.

Table 3. Tumor response by RECIST ver. 1.1

\begin{tabular}{lc} 
Best overall response & No. (\%) \\
Complete response & $4(3.8)$ \\
Partial response & $5(4.8)$ \\
Stable disease & $22(21.2)$ \\
Progressive disease & $70(67.3)$ \\
Not assessed & $3(2.9)$ \\
\hline
\end{tabular}

RECIST, Response Evaluation Criteria in Solid Tumor.

\section{Efficacy}

Tumor response was evaluated in 101 of 104 patients. Four patients $(3.8 \%)$ showed complete response (CR) and five patients $(4.8 \%)$ showed a partial response (PR) by RECIST. The overall response rate $(\mathrm{ORR}=\mathrm{CR}+\mathrm{PR})$ and disease control rate $(\mathrm{DCR}=\mathrm{CR}+\mathrm{PR}+$ stable disease $)$ was $8.6 \%$ and $29.8 \%$, respectively (Table 3 ). The median follow-up duration was 7.1 months (95\% confidential interval [CI], 5.96 to 8.30), and 85 patients $(81.7 \%)$ had progression of disease and 30 patients $(28.8 \%)$ had died by the time of this analysis. The median PFS was 2.73 months (95\% CI, 2.67 to 2.85) in all patients (Fig. 1A), and significantly longer survival was observed for patients with disease control $(\mathrm{CR}+\mathrm{PR}+$ stable disease) compared to those with progressed disease (PD) (median PFS, 5.4 months vs. 2.2 months; $\mathrm{p}<0.001$ ) (Fig. 1B and C).

\section{Association between baseline characteristics and efficacy}

There was no difference in ORR, DCR, PFS, or OS according to melanoma subtypes (Fig. 1D and E). Pretreatment median ANC was significantly higher in patients with PD compared to those who achieved disease control $(4,017$ cells $/ \mu \mathrm{L}$ vs. 3,183 cells $/ \mu \mathrm{L}, \mathrm{p}=0.016)$, while pretreatment median ALC was significantly lower in patients with PD compared to those who achieved disease control $(1,530$ cells $/ \mu \mathrm{L}$ vs. 1,833 cells $/ \mu \mathrm{L}, \mathrm{p}=0.049)$. When ANC and ALC were incorporated in the NLR, significantly higher NLR was observed in patients who experienced PD than in those with disease control (2.63 vs. 1.90, $\mathrm{p}=0.003$ ) (Table 4 ). In addition, we found that PFS and OS were significantly longer in patients with low NLR compared to those with high NLR (median PFS, 2.8 vs. 1.3 months; $\mathrm{p}<0.001$ and median OS, not reached vs. 2.6 months; $p<0.001$ ) (Fig. 1F and G). In univariate analysis between baseline characteristics and survival, ECOG PS, AJCC M stage, liver metastasis, and NLR showed significant association with PFS, and ECOG PS and NLR showed significant association with OS. There was no difference in PFS and OS according to the presence of brain metastasis, BRAF mutation, $c$-KIT mutation, and LDH elevation before treatment, or the number of previous therapies. In multivariate analysis, ECOG PS, liver metastasis, and NLR were independent prognostic factors for PFS, and ECOG PS and liver metastasis were independent prognostic factors for OS (Table 5). For selection of patients likely to benefit from ipilimumab, the patients were divided into three groups based on independent prognostic factors for PFS and OS, 
A

B
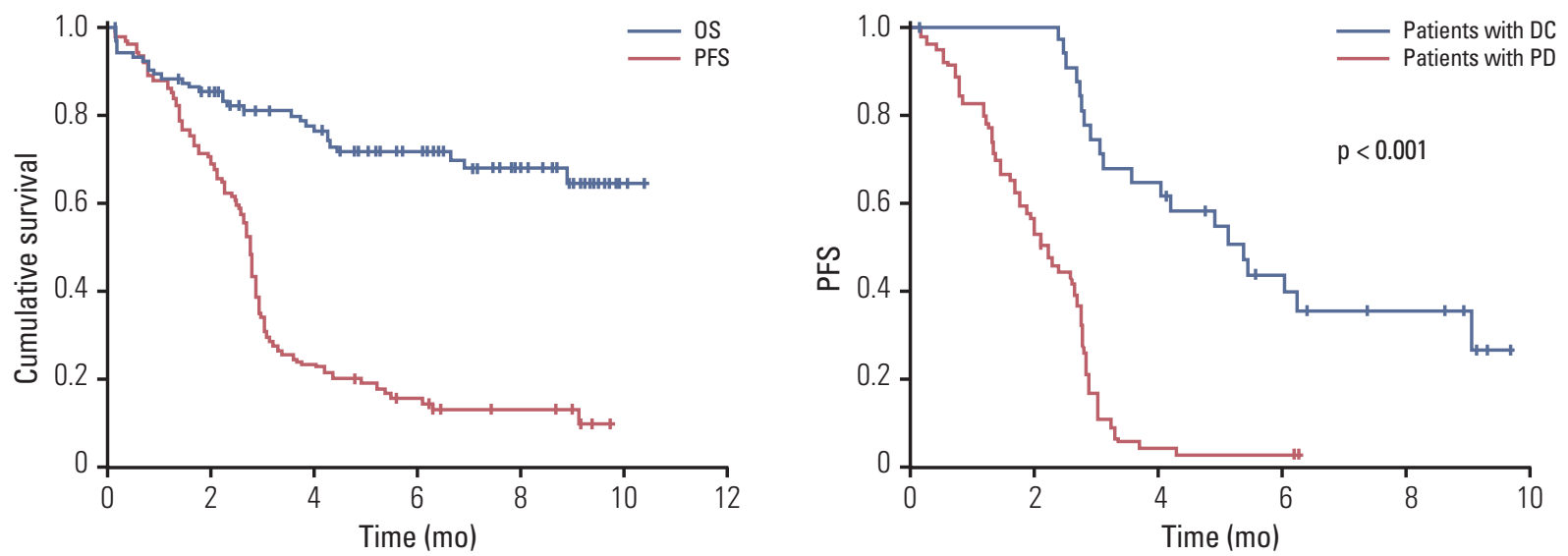

C
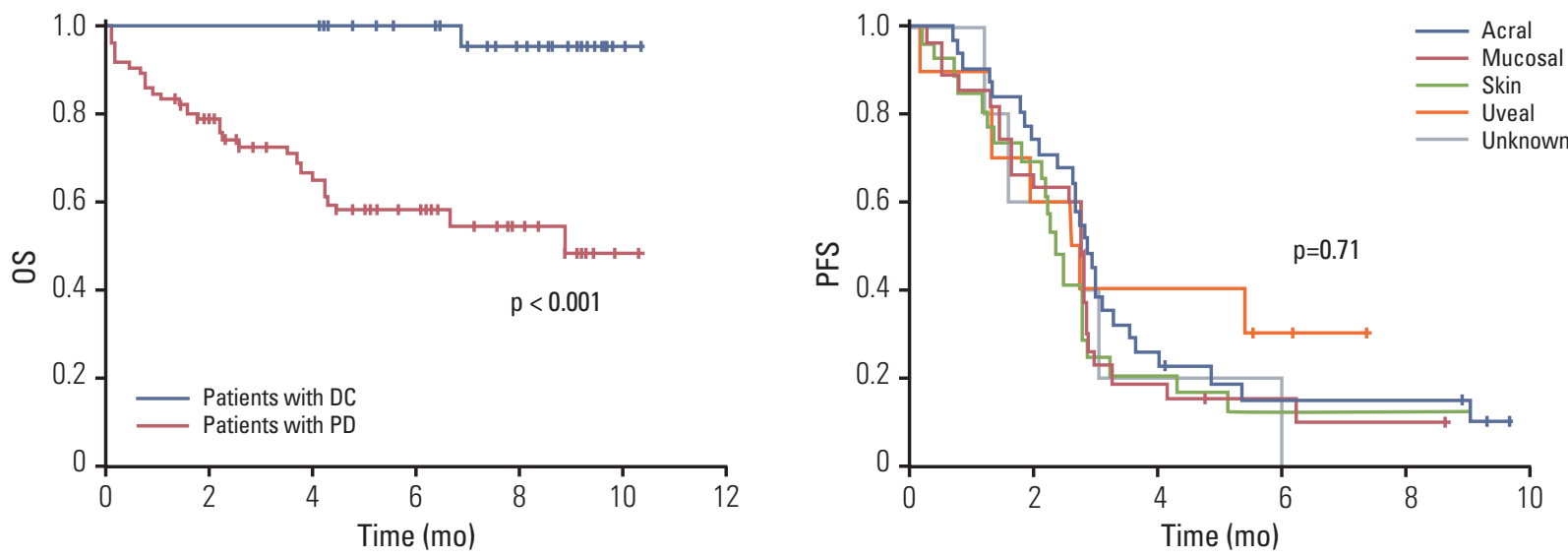

$\mathbf{E}$
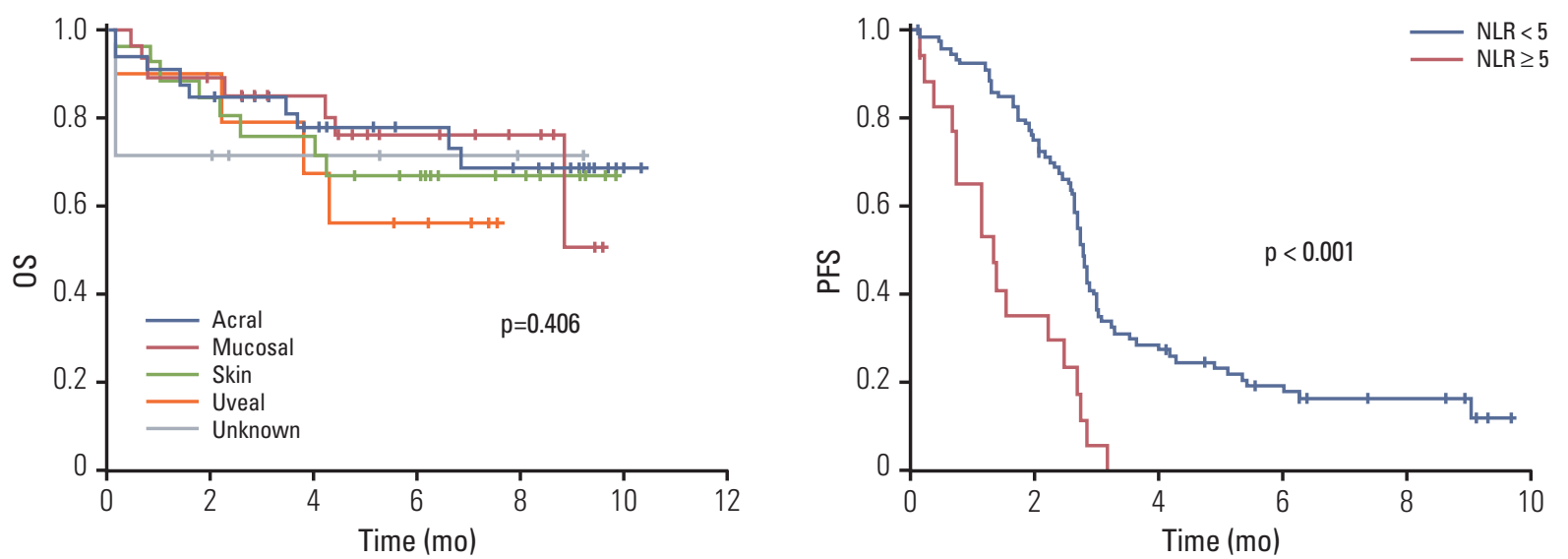

Fig. 1. Kaplan-Meier curves. (A) Progression-free survival (PFS) and overall survival (OS) in all patients. (B) PFS according to response. (C) OS according to response. (D) PFS according to primary site. (E) OS according to primary site. (F) PFS according to according to neutrophil to lymphocyte ratio (NLR). (G) OS according to according to NLR. (H) PFS according to risk factors (Eastern Cooperative Oncology Group performance status [ECOG PS] [0-1 vs. 2], liver metastasis [no vs. yes], and NLR [ $<5$ or $\geq 5$ ]). (I) OS according to risk factors (ECOG PS [0-1 vs. 2] and liver metastasis [no vs. yes]). DC, disease control (complete response+partial response+stable disease); PD, progression of disease. (Continued to the next page) 
G
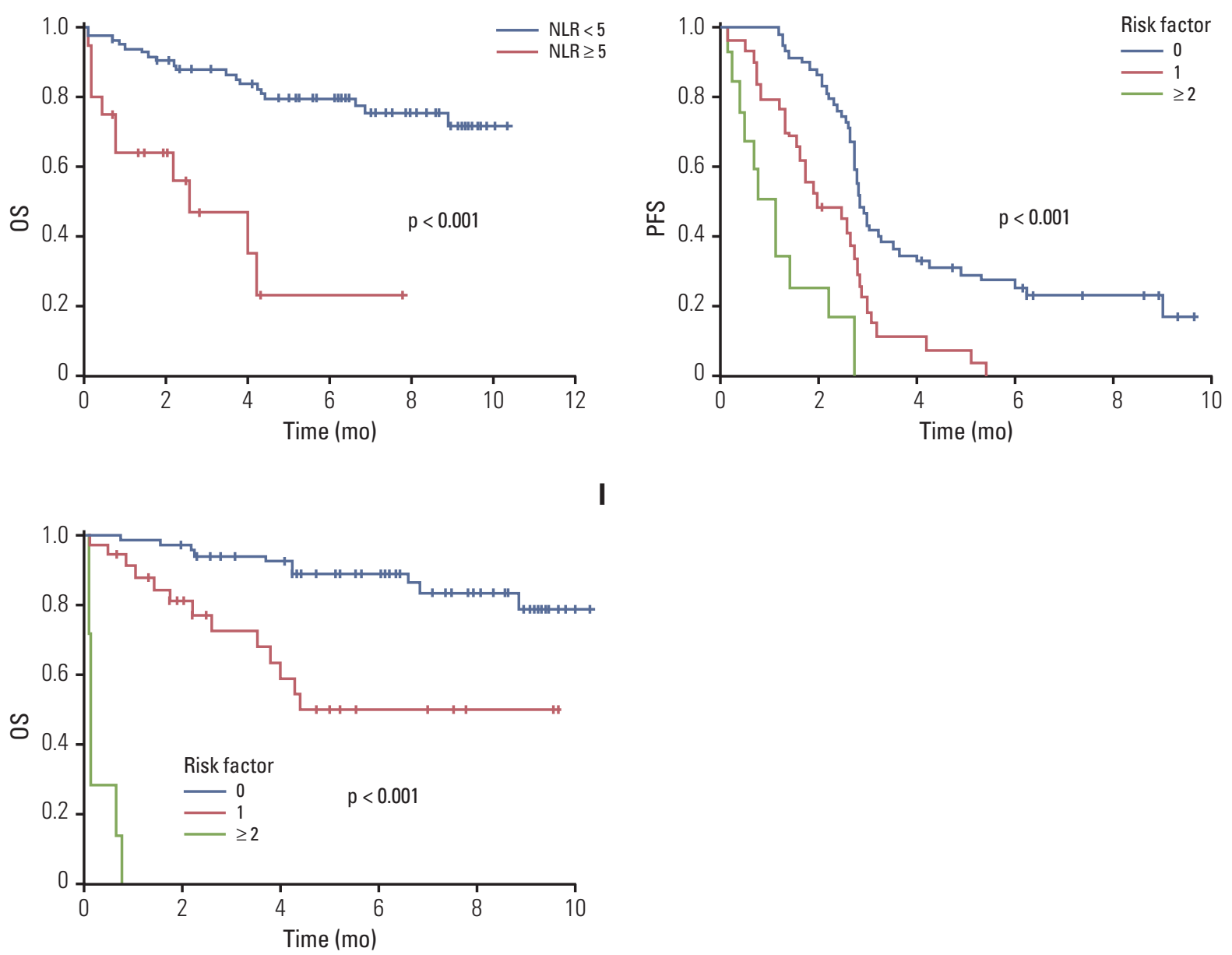

Fig. 1. (Continued from the previous page)

Table 4. Blood parameters at baseline according to on-treatment response to ipilimumab

\begin{tabular}{|c|c|c|c|c|c|c|c|c|}
\hline Response & $\begin{array}{l}\text { WBC count } \\
(\text { cells } / \mu \mathrm{L})\end{array}$ & p-value & $\begin{array}{c}\text { ANC } \\
\text { (cells/ } / \mathrm{L})\end{array}$ & p-value & $\begin{array}{l}\text { ALC count } \\
\text { (cells/ } \mu \mathrm{L})\end{array}$ & p-value & NLR & p-value \\
\hline $\mathrm{CR}+\mathrm{PR}+\mathrm{SD}$ & $5,320(4,950-6,670)$ & 0.076 & $3,183(2,480-3,742)$ & 0.016 & $1,833(1,368-2,468)$ & 0.049 & $1.90(1.24-2.45)$ & 0.003 \\
\hline PD & $6,140(5,230-8,750)$ & & $4,017(2,863-5,784)$ & & $1,530(1,110-1,955)$ & & $2.63(1.76-5.14)$ & \\
\hline
\end{tabular}

Values are presented as median (interquartile range). WBC, white blood cell; ANC, absolute neutrophil count; ALC, absolute lymphocyte count; NLR, neutrophil to lymphocyte ratio; CR, complete response; PR, partial response; SD, stable disease; $\mathrm{PD}$, progressed disease. 


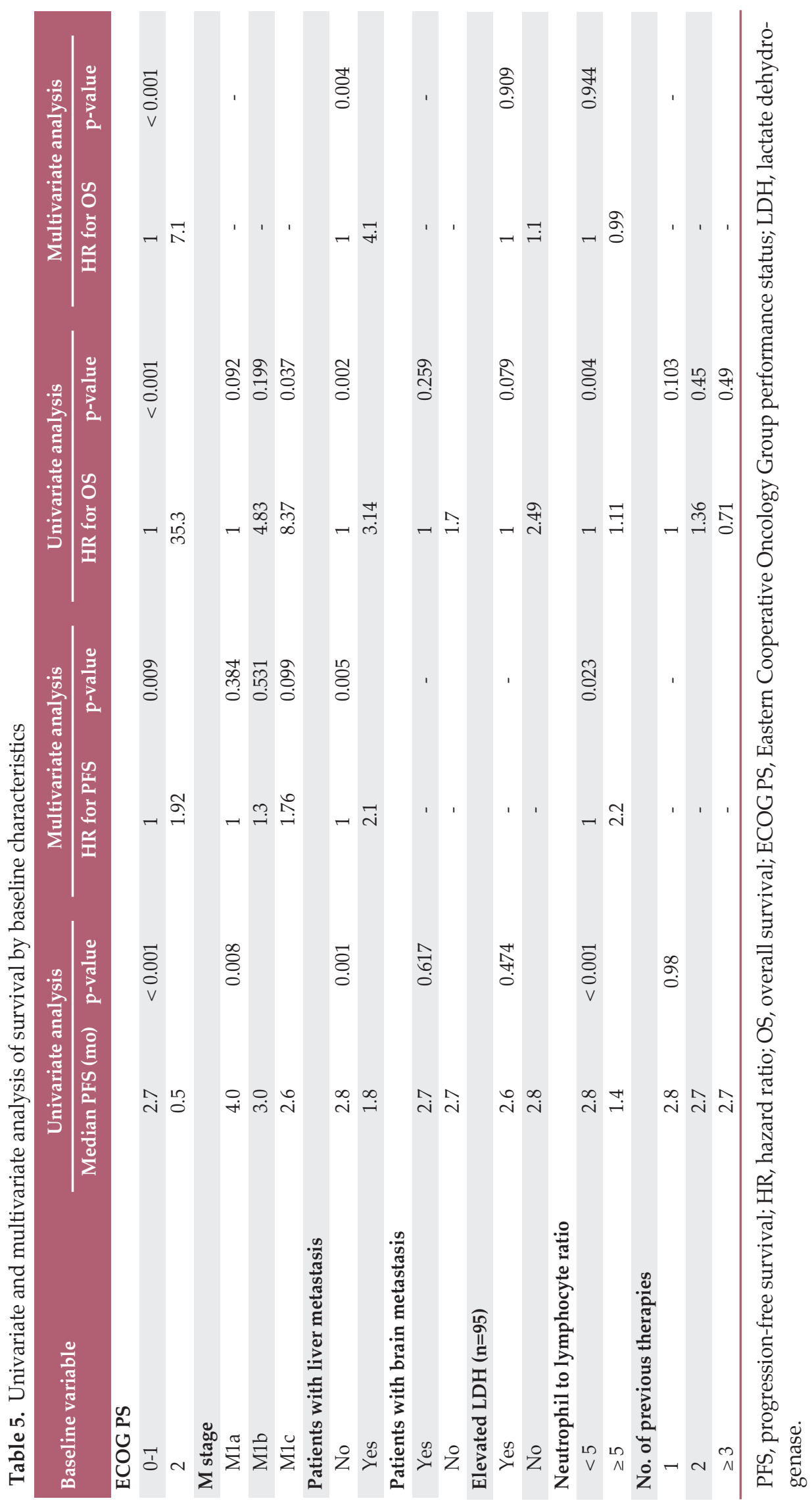


respectively. Patients without adverse factors had longer PFS and OS than patients with adverse factors (Fig. $1 \mathrm{H}$ and I).

\section{Discussion}

Ipilimumab improved survival in several clinical trials and many EAPs in pretreated and treatment-naïve patients with advanced melanoma $[3,7,8]$. However, due to low disease incidence, clinical trials and EAPs with ipilimumab have excluded Asian patients with advanced melanoma, therefore efficacy and safety data on ipilimumab in the Asian patient population has been limited. We report outcomes with ipilimumab in a real-world routine clinical setting in Asian patients with different melanoma subtypes compared to Western patients. Although the median duration of followup was 7 months, ipilimumab showed consistent efficacy compared to Western patients with advanced melanoma [3,7,8,14,15].

Malignant melanoma has demographic and ethnic differences. It is the fifth most common cancer in the United States; however, its incidence is relatively rare among Africans, Hispanics, and Asians $[1,16]$. The histologic subtype also differs, with the most common histology being superficial spreading melanoma in whites and acral lentiginous melanoma among blacks [17]. According to recent data, the most common primary site in Asians [9-12] was acral lentiginous melanoma. Mucosal melanoma is the second most common primary site in patients in China and Korea [10,11], although it is extremely rare in Caucasians [18]. In patients from Western countries, there were no reported differences in efficacy of ipilimumab in patients with primary acral and mucosal melanoma compared with cutaneous melanoma $[8,19]$. In the current study, the most common subtype was acral and mucosal melanoma (57.7\%) with no survival differences according to subtypes.

Although the follow up duration was not long, the median OS was not yet reached and the median PFS was 2.73 months (95\% CI, 2.67 to 2.85). These results are comparable to those of Western patients treated with ipilimumab $[3,7,8,14,15]$, and the ORR of $8.6 \%$ and DCR of $29.8 \%$ were similar to EAP data in Western countries $[3,7,8,14,15]$.

Only two small studies of ipilimumab in Asians have been reported. In a prospective phase II trial in 20 previously untreated Japanese patients, the median PFS was 2.74 months [20]. The second study was an EAP study in 31 previously treated Taiwanese patients, which reported a PFS of 4.1 months [21]. Therefore, ipilimumab is a feasible treatment option for Asian patients with metastatic melanoma.

For identification of predictive markers of efficacy for ipil- imumab, we analyzed the association between the survival and baseline characteristics including blood parameters. Several blood parameters, including LDH, ANC, ALC, NLR, and $\mathrm{C}$-reactive protein, have been suggested as associated markers with efficacy of ipilimumab $[8,15,19,22-24]$. In univariate analysis, ECOG PS, AJCC M stage, liver metastasis, and NLR showed significant association with PFS, and ECOG PS and NLR showed significant association with OS. There was no difference in PFS and OS according to brain metastasis or the number of previous therapies. In multivariate analysis, ECOG PS, liver metastasis, and NLR were independent prognostic factors for PFS and ECOG PS and liver metastasis were independent prognostic factors for OS. In addition, patients without adverse factors had longer survival than those with adverse factors. Therefore, ipilimumab could be administered in patients with good PS, lower NLR, and no liver metastasis.

The safety of ipilimumab in the current study was similar to that reported in patients with cutaneous melanoma [25]. There were no treatment-related deaths or withdrawal from the study due to drug-related AEs. The most frequent toxicities were immune-related skin toxicities. Despite development of colitis in seven patients ( $6.7 \%)$, there were no grade 3 or higher severe cases, and there was no immune related hepatitis. One patient with multiple liver metastases had elevated AST / ALT of grade 3 after two cycles of ipilimumab, and computed tomography scan showed a further increase in size and numerous liver metastatic nodules and masses, replacing almost the entire liver. Therefore, we considered that the hepatitis was related to disease progression, not ipilimumab. Twenty-two patients $(20.3 \%)$ suffered from fatigue; however, these patients had poor PS (ECOG PS 1 or 2) or heavy tumor burden at the baseline, thus this symptom could be disease-related.

There are several limitations in this study, which are common in retrospective cohort studies, including potential patient selection bias, lack of strict methods of timing of assessments, and some missing data of toxicity profiles. Among 104 patients, 69 patients $(66.4 \%)$ received all four doses of ipilimumab, and 15 patients completed only one treatment cycle. The most common reason for discontinuation of ipilimumab was rapid disease progression, because some patients with severe tumor burden who did not have other treatment options had to wait for approval of the NPP before receiving treatment with ipilimumab. Despite these limitations, because the NPP population included patients who would have been excluded from a clinical trial due to their poor medical condition, our result may be more representative of advanced melanoma patients in the clinical setting. In the Korean melanoma patients, ipilimumab showed a similar efficacy and tolerability compared with Western patients, regardless of subtypes. All subtypes should benefit 
from ipilimumab with consideration of PS, liver metastasis, and NLR.

\section{Conclusion}

The current NPP cohort in Korean patients with advanced melanoma showed that ipilimumab monotherapy had a manageable safety profile and antitumor activity. These findings are consistent with the safety and activity profile of ipilimumab in Asian patients who had mainly acral and mucosal melanoma compared to patients with cutaneous melanoma from Western studies.

\section{Conflicts of Interest}

The NPP was sponsored by Bristol-Myers Squibb.

\section{Acknowledgments}

We would like to thank the patients and investigators who participated in the Korean NPP.

\section{References}

1. Siegel RL, Miller KD, Jemal A. Cancer statistics, 2015. CA Cancer J Clin. 2015;65:5-29.

2. Dummer R, Hauschild A, Lindenblatt N, Pentheroudakis G, Keilholz U; ESMO Guidelines Committee. Cutaneous melanoma: ESMO Clinical Practice Guidelines for diagnosis, treatment and follow-up. Ann Oncol. 2015;26 Suppl 5:v126-32.

3. Ascierto PA, Simeone E, Sileni VC, Pigozzo J, Maio M, Altomonte $\mathrm{M}$, et al. Clinical experience with ipilimumab 3 $\mathrm{mg} / \mathrm{kg}$ : real-world efficacy and safety data from an expanded access programme cohort. J Transl Med. 2014;12:116.

4. Tsao H, Atkins MB, Sober AJ. Management of cutaneous melanoma. N Engl J Med. 2004;351:998-1012.

5. Hodi FS, O'Day SJ, McDermott DF, Weber RW, Sosman JA, Haanen JB, et al. Improved survival with ipilimumab in patients with metastatic melanoma. N Engl J Med. 2010;363: 711-23.

6. Chapman PB, Hauschild A, Robert C, Haanen JB, Ascierto P, Larkin J, et al. Improved survival with vemurafenib in melanoma with BRAF V600E mutation. N Engl J Med. 2011; 364:2507-16.

7. Schadendorf D, Hodi FS, Robert C, Weber JS, Margolin K, Hamid $\mathrm{O}$, et al. Pooled analysis of long-term survival data from phase II and phase III trials of ipilimumab in unresectable or metastatic melanoma. J Clin Oncol. 2015;33:188994.

8. Ahmad SS, Qian W, Ellis S, Mason E, Khattak MA, Gupta A, et al. Ipilimumab in the real world: the UK expanded access programme experience in previously treated advanced melanoma patients. Melanoma Res. 2015;25:432-42.

9. Ishihara K, Saida T, Otsuka F, Yamazaki N; Prognosis and Statistical Investigation Committee of the Japanese Skin Cancer Society. Statistical profiles of malignant melanoma and other skin cancers in Japan: 2007 update. Int J Clin Oncol.
2008;13:33-41.

10. Jang HS, Kim JH, Park KH, Lee JS, Bae JM, Oh BH, et al. Comparison of melanoma subtypes among Korean patients by morphologic features and ultraviolet exposure. Ann Dermatol. 2014;26:485-90.

11. Chi Z, Li S, Sheng X, Si L, Cui C, Han M, et al. Clinical presentation, histology, and prognoses of malignant melanoma in ethnic Chinese: a study of 522 consecutive cases. BMC Cancer. 2011;11:85.

12. Chang JW, Yeh KY, Wang CH, Yang TS, Chiang HF, Wei FC, et al. Malignant melanoma in Taiwan: a prognostic study of 181 cases. Melanoma Res. 2004;14:537-41.

13. Balch CM, Gershenwald JE, Soong SJ, Thompson JF, Atkins MB, Byrd DR, et al. Final version of 2009 AJCC melanoma staging and classification. J Clin Oncol. 2009;27:6199-206.

14. Kelderman S, Heemskerk B, van Tinteren $H$, van den Brom RR, Hospers GA, van den Eertwegh AJ, et al. Lactate dehydrogenase as a selection criterion for ipilimumab treatment in metastatic melanoma. Cancer Immunol Immunother. 2014;63: 449-58.

15. Berrocal A, Arance A, Lopez Martin JA, Soriano V, Munoz E, Alonso L, et al. Ipilimumab for advanced melanoma: experience from the Spanish Expanded Access Program. Melanoma Res. 2014;24:577-83.

16. Cormier JN, Xing Y, Ding M, Lee JE, Mansfield PF, Gershenwald JE, et al. Ethnic differences among patients with cutaneous melanoma. Arch Intern Med. 2006;166:1907-14.

17. Wu XC, Eide MJ, King J, Saraiya M, Huang Y, Wiggins C, et al. Racial and ethnic variations in incidence and survival of cutaneous melanoma in the United States, 1999-2006. J Am Acad Dermatol. 2011;65(5 Suppl 1):S26-37.

18. Seetharamu N, Ott PA, Pavlick AC. Mucosal melanomas: a case-based review of the literature. Oncologist. 2010;15:772-81. 
19. Del Vecchio M, Di Guardo L, Ascierto PA, Grimaldi AM, Sileni VC, Pigozzo J, et al. Efficacy and safety of ipilimumab $3 \mathrm{mg} / \mathrm{kg}$ in patients with pretreated, metastatic, mucosal melanoma. Eur J Cancer. 2014;50:121-7.

20. Yamazaki N, Kiyohara Y, Uhara H, Fukushima S, Uchi H, Shibagaki N, et al. Phase II study of ipilimumab monotherapy in Japanese patients with advanced melanoma. Cancer Chemother Pharmacol. 2015;76:997-1004.

21. Wu CF, Huang WK, Liu JH, Lin CY, Liu TC, Lin CC, et al. Ipilimumab in treating Asian patient with advanced melanoma: the preliminary report of Taiwan ipilimumab extended access program group. J Clin Oncol. 2015;33(Suppl):Abstr e20069.

22. Ferrucci PF, Gandini S, Battaglia A, Alfieri S, Di Giacomo AM, Giannarelli D, et al. Baseline neutrophil-to-lymphocyte ratio is associated with outcome of ipilimumab-treated metastatic melanoma patients. Br J Cancer. 2015;112:1904-10.

23. Delyon J, Mateus C, Lefeuvre D, Lanoy E, Zitvogel L, Chaput $\mathrm{N}$, et al. Experience in daily practice with ipilimumab for the treatment of patients with metastatic melanoma: an early increase in lymphocyte and eosinophil counts is associated with improved survival. Ann Oncol. 2013;24:1697-703.

24. Queirolo P, Spagnolo F, Ascierto PA, Simeone E, Marchetti P, Scoppola A, et al. Efficacy and safety of ipilimumab in patients with advanced melanoma and brain metastases. J Neurooncol. 2014;118:109-16.

25. Weber JS, Kahler KC, Hauschild A. Management of immunerelated adverse events and kinetics of response with ipilimumab. J Clin Oncol. 2012;30:2691-7. 